\title{
Design and Simulation of Direct and Indirect Back EMF Sliding Mode Observer for Sensorless Control of PMSM
}

Research Article

\author{
Viktor Petro*, Karol Kyslan \\ Technical University of Košice, Faculty of Electrical Engineering and Infor-matics, Letná 9, 042 00, Košice, Slovak Republic
}

Received: November 13, 2020; Accepted: November 27, 2020

\begin{abstract}
This article compares two sensorless control algorithms for a permanent magnet synchronous machine (PMSM) based on the back-EMF sliding mode observer (SMO). Indirect SMO (I-SMO) treats the back-EMF voltages as a disturbance. Direct SMO (D-SM0) considers back-EMF voltages as state-space variables. The same phase-locked loop (PLL) is used for both observers for extraction of the rotor position and speed values from the observed back-EMF voltages. In a sensorless control, the observed speed is used as feedback for the PI controller, and the observed position is used in the Park transformations. Both observers have been implemented and tested with standard field-oriented control. Simulation results indicate rather comparable speed and position estimation precision for both, but the D-SMO indicates slightly higher precision in steady-state. Even more, a tuning procedure of the D-SMO is more straightforward when compared to the I-SMO. Thus, the D-SMO was further verified experimentally with the OP 5600 rapid prototyping device and with a 350 W PMSM drive. Experimental results of the D-SM0 are included at the end of the paper.
\end{abstract}

Keywords: back-EMF sliding mode observer • sensorless control • PMSM • phase-locked loop

\section{Introduction}

Nowadays the permanent magnet synchronous motor (PMSM) is widely used in industry. The main advantages of using PMSM are high power density and efficiency. Most of the time, a field-oriented control (FOC) is applied to achieve precise and smooth torque control. A reliable and accurate rotor shaft position information is required to achieve this purpose, which can be obtained using resolvers or encoders mounted on the rotor shaft. These sensors increase the overall cost and decrease mechanical reliability. Therefore, several approaches have been developed to eliminate position sensors and to achieve cost reduction and system robustness. Further, software development and testing costs must be considered.

One of the widely used approaches for sensorless PMSM control is the back electromotive force (EMF) estimation where information of the rotor position can be further extracted from the back-EMF value. The magnitude of the back-EMF value cannot be directly measured during the motor control, and therefore its estimation is required. Several types of observers can be used for this purpose, such as Kalman filter (Horváth and Kuslits, 2019; Kyslan et al., 2019), Luenberger observer (Tarczewski et al., 2018) or Sliding Mode Observer (SMO). The approach which makes use of back-EMF estimation is reliable approximately above $10 \%$ of the nominal rotor speed. The extraction of the rotor position from the back-EMF is done in most cases via a trigonometric function or by using a phaselocked loop (PLL).

There is a wide range of different SMO for back-EMF estimation, but in general, they can be classified into two main categories (Changsheng and Elbuluk, 2002). If the back-EMF is considered as a disturbance and if it is identified by matching the estimated currents with the measured currents using a second-order observer, then the 


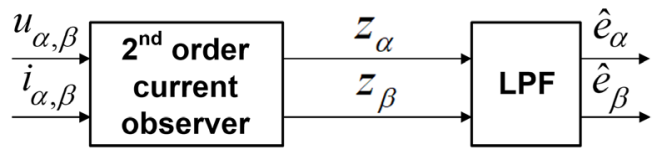

Fig. 1. Block diagram of indirect SMO (Changsheng and Elbuluk, 2002).

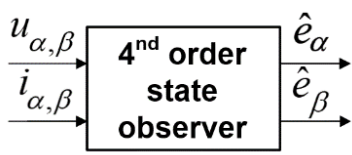

Fig. 2. Block diagram of direct SMO (Changsheng and Elbuluk, 2002)

observer is categorised as indirect and it is often referred to as a reduced-order observer (Chi, 2007; Chi et al., 2009; Elbuluk and Changsheng, 2003; Texas Instruments, 2001; Utkin et al., 1999; Zambada, 2010). Hereinafter we will reference it as indirect SMO (I-SMO) and its variables are shown in Figure 1.

If the back-EMF is considered as a state variable, then the observer is categorised as direct (Freescale semiconductor, 2008; Wang et al., 2013) or (Chen et al., 2000). In this case, a fourth-order observer is used for direct back-EMF estimation and is often referred to as a full-order observer. Hereafter we will refer to it as direct SMO (D-SMO) and its variables are shown in Figure 2.

Both observers consider input command voltages as $u_{\alpha^{\prime}}, u_{\beta}$ and measured currents as $i_{\alpha^{\prime}} i_{\beta}$. In both cases, the outputs are the $\alpha$ and $\beta$ components of the estimated back-EMF $\hat{e}_{\alpha^{\prime}}, \hat{e}_{\beta}$. These components are further used for rotor position extraction. A typical characteristic of the I-SMO is a low-pass filter (LPF) which is used to filter out the high-frequency components from the outputs of the discontinuous control $z_{\alpha^{\prime}} z_{\beta}$. Variables $z_{\alpha^{\prime}} z_{\beta}$ will be defined later in the text.

\section{Design of the Indirect Sliding Mode Observer (I-SMO)}

The mathematical model of PMSM in the $\alpha, \beta$ reference frame is commonly used for position observer construction. The mathematical model for the surface-mounted permanent magnet synchronous motor (SMPMSM) is given below, in which stator current components are state variables and are written as:

$$
\left[\begin{array}{c}
\dot{i}_{\alpha} \\
\dot{i}_{\beta}
\end{array}\right]=\frac{1}{L_{s}}\left[\begin{array}{cc}
-R_{s} & 0 \\
0 & -R_{s}
\end{array}\right]\left[\begin{array}{c}
i_{\alpha} \\
i_{\beta}
\end{array}\right]+\frac{1}{L_{s}}\left[\begin{array}{c}
u_{\alpha}-e_{\alpha} \\
u_{\beta}-e_{\beta}
\end{array}\right],
$$

where $R_{s}=R_{2 p h} / 2$ and $R_{2 p h}$ is the resistance between two phases, $L_{s}=L_{2 p h} / 2$ and $L_{2 p h}$ is the inductance between two phases, $e_{\alpha}=-E_{e x} \sin \left(\theta_{e}\right)$ and $e_{\beta}=E_{e x} \cos \left(\theta_{e}\right)$ are the back-EMF components, where $\theta_{e}$ is the electrical rotor position, $E_{e x}=\omega_{e} \lambda_{P M}$ is the extended EMF, where $\omega_{e}$ is the electrical angular velocity and $\lambda_{P M}=\frac{2}{3} \frac{k_{t}}{p}=\frac{k_{e}}{p}$ is the permanent magnet flux, where $k_{t}$ is the motor torque constant, $k_{e}$ is the EMF constant and $p$ is the motor pole pairs number.

One of the first applications of the SMO for sensorless SMPMSM control can be found in Utkin et al. (1999). A 2nd order current observer was used for observing the components of the back-EMF and another observer (refer to as Kalman filter) was used to extract the rotor position value from the observed back-EMF components. The disadvantage of this solution was its complexity and slow dynamic response of the Kalman filter as discussed in Changsheng and Elbuluk (2002) or Elbuluk and Changsheng (2003). The next-generation of commercially used SMO observers introduced a feedback from the observed back-EMF components and avoided using the Kalman filter. A trigonometric function can be used instead of the Kalman filter for the rotor position extraction from the observed back-EMF values (Texas Instruments, 2001; Zambada, 2010) or, the PLL circuit was proposed as an alternative solution in Chi et al. (2009) and Wang et al. (2020) in an attempt to cause further improvement of the rotor position extraction. In this paper, the observer like the one in Chi et al. (2009) was studied. From the sliding mode theory, the switching function is defined as follows: 


$$
\mathbf{s}(\mathbf{x})=\left[\begin{array}{c}
\bar{i}_{\alpha} \\
\bar{i}_{\beta}
\end{array}\right]=\left[\begin{array}{c}
\hat{i}_{\alpha}-i_{\alpha} \\
\hat{i}_{\beta}-i_{\beta}
\end{array}\right],
$$

where superscript ' $"$ indicates an observed parameter. The switching surface (or the so-called sliding surface hyperplane) is defined when the following condition is met:

$$
\mathbf{s}(\mathbf{x})=0 .
$$

It means that the phase plane is divided into two regions where the switching function $\mathbf{s}(\mathbf{x})$ has different signs. According to the sign of the switching function $\mathbf{s}(\mathbf{x})$, the observer state trajectories are forced towards the sliding hyperplane. In ideal SMO, the sign of the switching function $\mathbf{s}(\mathbf{x})$ is verified with infinite frequency and the state trajectories reach and stay on the sliding hyperplane, i.e. $\mathbf{s}(\mathbf{x})=0$ and $\dot{\mathbf{s}}(\mathbf{x})=0$.

The matrix form of the observer equations is shown below:

$$
\left[\begin{array}{c}
\hat{i}_{\alpha} \\
\hat{i}_{\beta}
\end{array}\right]=\frac{1}{L_{s}}\left[\begin{array}{cc}
-R_{s} & 0 \\
0 & -R_{s}
\end{array}\right]\left[\begin{array}{c}
\hat{i}_{\alpha} \\
\hat{i}_{\beta}
\end{array}\right]+\frac{1}{L_{s}}\left[\begin{array}{c}
u_{\alpha}-l \hat{e}_{\alpha}-z_{\alpha} \\
u_{\beta}-l \hat{e}_{\beta}-z_{\beta}
\end{array}\right],
$$

$\hat{e}_{\alpha}, \hat{e}_{\beta}$ are the observed components of the back-EMF and also a feedback for the SMO. They can be obtained by filtering the output of the discontinuous control $z_{\alpha}, z_{\beta}$ using a low-pass filter (LPF):

$$
\left[\begin{array}{c}
\hat{e}_{\alpha} \\
\hat{e}_{\beta}
\end{array}\right]=\frac{\omega_{c}}{s+\omega_{c}}\left[\begin{array}{c}
z_{\alpha} \\
z_{\beta}
\end{array}\right],
$$

where $\omega_{c}$ is the cut-off frequency of the filter. For the output of the discontinuous control $z_{\alpha}, z_{\beta}$ will be the following:

$$
\left[\begin{array}{c}
z_{\alpha} \\
z_{\beta}
\end{array}\right]=k_{1}\left[\begin{array}{c}
\operatorname{sign}\left(\hat{i}_{\alpha}-i_{\alpha}\right) \\
\operatorname{sign}\left(\hat{i}_{\beta}-i_{\beta}\right)
\end{array}\right],
$$

where the parameter $k_{1}>0$ is the switching gain of the SMO obtained from Lyapunov stability analysis. According to the analysis in Chi (2007), $k_{1}$ should be calculated as:

$$
k_{1}(1+l)>\max \left(\left|e_{\alpha}\right|,\left|e_{\beta}\right|\right),
$$

where $l$ is the feedback gain of the SMO and must be greater than -1 according to Eq. (7), if $k_{1}>0$ and the product of $k_{1}$ and $(1+l)$ is larger than the maximum amplitude of the back-EMF components. The dynamic model of the SMO can be obtained by subtracting Eq. (1) from Eq. (4):

$$
\begin{aligned}
& \dot{\dot{i}_{\alpha}}=-\frac{R_{s}}{L_{s}} \bar{i}_{\alpha}-\frac{1}{L_{s}}\left(l \hat{e}_{\alpha}-e_{\alpha}\right)-\frac{1}{L_{s}} z_{\alpha}, \\
& \dot{\dot{i}_{\beta}}=-\frac{R_{s}}{L_{s}} \bar{i}_{\beta}-\frac{1}{L_{s}}\left(l \hat{e}_{\beta}-e_{\beta}\right)-\frac{1}{L_{s}} z_{\beta} .
\end{aligned}
$$

If $k$ is positively large enough to guarantee that:

$$
\dot{\mathbf{s}}(\mathbf{x})^{\mathrm{T}} \mathbf{s}(\mathbf{x})<0,
$$

then the system trajectories are forced towards the hyperplane described in Eq. (3) and will reach it. After the system is in sliding mode, the estimated currents will converge to the real measured currents and therefore $\bar{i}_{\alpha}$ and 
$\bar{i}_{\beta}$ become zero, and so will their derivatives. After substituting the above mentioned into Eq. (8) and considering that the system is in the ideal sliding mode, we get:

$$
\left[\begin{array}{c}
e_{\alpha} \\
e_{\beta}
\end{array}\right]=(1+l)\left[\begin{array}{c}
\hat{e}_{\alpha} \\
\hat{e}_{\beta}
\end{array}\right] .
$$

Equation (10) shows the relationship between the real back-EMF and the observed one from the SMO. If the feedback gain is $0>l>-1$, then the amplitude of the observed back-EMF is larger than the real value. Since the rotor position is obtained from the augmented observed back-EMF value, the proposed SMO is capable of performing sensorless operation at lower speeds provided the feedback parameter is chosen from the mentioned interval. On the other hand, at high-speed operation, faster convergence of the estimated errors to zero is required. Setting the feedback gain $l=1$ after a predefined speed threshold will guarantee a higher convergence rate of $\mathbf{s}(\mathbf{x})$ to zero. Therefore, feedback gain $l$ can be adapted to the speed, and a better performance of the SMO can be achieved at higher speeds by increasing the value of $l$.

Since the signum function is executed with finite frequency in real life, the well-known chattering phenomenon will occur. So, a saturation function will be used in simulation to suppress this issue, instead of the discontinuous signum function, similarly as in Chi et al. (2009).

The electrical rotor position extraction from the observed back-EMF components as well as the electrical angular velocity determination was done via the PLL circuit. The design of the PLL will be discussed in Section 4 as it is the same for I-SMO and D-SMO. The overall block diagram of the I-SMO with the PLL can be seen in Fig. 3

\section{Design of the Direct Sliding Mode Observer (D-SM0)}

The back-EMF components are considered as state variables for the D-SMO. These components can be state variables under the assumption that the mechanical time constant is many times larger than the electrical time constant of the drive. This is a well-known infinite inertia approach, where $\dot{\omega} \approx 0$ is assumed. Under this approach, the derivatives of the back-EMF components can be expressed as follows:

$$
\left[\begin{array}{c}
\dot{e}_{\alpha} \\
\dot{e}_{\beta}
\end{array}\right]=\omega_{e}\left[\begin{array}{c}
-e_{\beta} \\
e_{\alpha}
\end{array}\right]
$$

The extended mathematical model for the SMPMSM is also referred to as a full-order mathematical model and can be written as:

$$
\left[\begin{array}{c}
\dot{\mathbf{i}} \\
\dot{\mathbf{e}}
\end{array}\right]=\left[\begin{array}{cc}
\mathbf{A}_{11} & \mathbf{A}_{12} \\
\mathbf{0} & \mathbf{A}_{22}
\end{array}\right]\left[\begin{array}{c}
\mathbf{i} \\
\dot{\mathbf{e}}
\end{array}\right]+\left[\begin{array}{c}
\mathbf{B}_{1} \\
\mathbf{0}
\end{array}\right] \mathbf{u},
$$

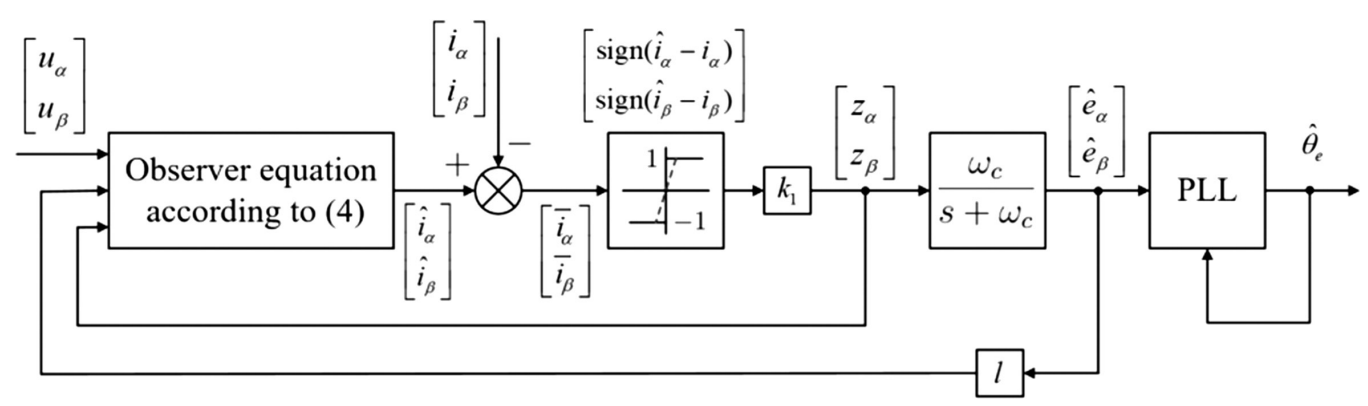

Fig. 3. Block diagram of the indirect SMO with PLL implementation. 
where:

$$
\begin{aligned}
& \mathbf{i}=\left[\begin{array}{ll}
i_{\alpha} & i_{\beta}
\end{array}\right]^{\mathrm{T}}, \quad \mathbf{e}=\left[\begin{array}{ll}
e_{\alpha} & e_{\beta}
\end{array}\right]^{\mathrm{T}}, \quad \mathbf{u}=\left[\begin{array}{ll}
u_{\alpha} & u_{\beta}
\end{array}\right]^{\mathrm{T}}, \\
& \mathbf{A}_{11}=a_{11} \mathbf{I}=-\frac{R_{s}}{L_{s}} \mathbf{I}, \quad \mathbf{A}_{12}=a_{12} \mathbf{I}=-\frac{1}{L_{s}} \mathbf{I}, \quad \mathbf{A}_{22}=a_{22} \mathbf{J}=\omega_{e} \mathbf{J}, \\
& \mathbf{B}_{1}=b_{1} \mathbf{I}=-a_{12} \mathbf{I}=\frac{1}{L_{s}} \mathbf{I}, \quad \mathbf{I}=\left[\begin{array}{ll}
1 & 0 \\
0 & 1
\end{array}\right], \quad \mathbf{J}=\left[\begin{array}{cc}
0 & -1 \\
1 & 0
\end{array}\right] .
\end{aligned}
$$

According to Freescale semiconductor (2008), the D-SMO (or full-order sliding mode observer) for SMPMSM is constructed as follows:

$$
\left[\begin{array}{c}
\dot{\hat{\mathbf{i}}} \\
\dot{\hat{\mathbf{e}}}
\end{array}\right]=\left[\begin{array}{cc}
\mathbf{A}_{11} & \mathbf{A}_{12} \\
\mathbf{0} & \hat{\mathbf{A}}_{22}
\end{array}\right]\left[\begin{array}{c}
\hat{\mathbf{i}} \\
\hat{\mathbf{e}}
\end{array}\right]+\left[\begin{array}{c}
\mathbf{B}_{1} \\
\mathbf{0}
\end{array}\right] \mathbf{u}+\mathbf{K}_{1}\left[\begin{array}{c}
\mathbf{I} \\
\mathbf{G}
\end{array}\right] \operatorname{sign}(\hat{\mathbf{i}}-\mathbf{i}),
$$

where $\hat{\mathbf{i}}=\left[\begin{array}{ll}\hat{i}_{\alpha} & \hat{i}_{\beta}\end{array}\right]^{\mathrm{T}}, \quad \hat{\mathbf{e}}=\left[\begin{array}{ll}\hat{e}_{\alpha} & \hat{e}_{\beta}\end{array}\right]^{\mathrm{T}}, \quad \hat{\mathbf{A}}_{22}=\hat{a}_{22} \mathbf{J}=\hat{\omega}_{e} \mathbf{J}, \quad \mathbf{G}=g_{1} \mathbf{I}+g_{2} \mathbf{J} \rightarrow$ feedback gain,

$$
\mathbf{K}_{1}=\left[\begin{array}{cccc}
k_{1} & 0 & 0 & 0 \\
0 & k_{1} & 0 & 0 \\
0 & 0 & k_{1} & 0 \\
0 & 0 & 0 & k_{1}
\end{array}\right] \rightarrow \text { switching gain. }
$$

The sliding hyperplane is chosen exactly as for the I-SMO (repeated here for the convenience of readers):

$$
\mathbf{s}=(\hat{\mathbf{i}}-\mathbf{i})=\mathbf{0} .
$$

In the case of the D-SMO, not only the current error vector s exists but also a back-EMF error vector which can be defined according to the following equation:

$$
\varepsilon=(\hat{\mathbf{e}}-\mathbf{e})
$$

Subtracting Eq. (12) from Eq. (13), the error equation is derived as follows (Chen et al., 2000):

$$
\left[\begin{array}{c}
\dot{\mathbf{s}} \\
\dot{\boldsymbol{\varepsilon}}
\end{array}\right]=\left[\begin{array}{cc}
\mathbf{A}_{11} & \mathbf{A}_{12} \\
\mathbf{0} & \mathbf{A}_{22}
\end{array}\right]\left[\begin{array}{l}
\mathbf{s} \\
\boldsymbol{\varepsilon}
\end{array}\right]+\mathbf{K}_{1}\left[\begin{array}{c}
\mathbf{I} \\
\mathbf{G}
\end{array}\right] \operatorname{sign}(\hat{\mathbf{i}}-\mathbf{i}) .
$$

The observer reaches sliding mode after the next condition is met:

$$
\mathbf{s}^{T} \dot{\mathbf{s}}<\mathbf{0}
$$

According to Eq. (16):

$$
\mathbf{s}^{T} \dot{\mathbf{s}}=\mathbf{s}^{T}\left(\mathbf{A}_{11} \mathbf{s}+\mathbf{A}_{12} \varepsilon\right)+\mathbf{s}^{T} \mathbf{K}_{1} \operatorname{Isign}(\mathbf{s}) .
$$

Knowing that:

$$
\mathbf{s}^{T} \operatorname{sign}(\mathbf{s})=\left[s_{\alpha} s_{\beta}\right] \operatorname{sign}\left[\begin{array}{c}
s_{\alpha} \\
s_{\beta}
\end{array}\right]=\left|s_{\alpha}\right|+\left|s_{\beta}\right|=|\mathbf{s}|,
$$


Equation (17) can be rewritten as follows:

$$
\begin{gathered}
\underbrace{\mathbf{s}^{T}\left(\mathbf{A}_{11} \mathbf{s}+\mathbf{A}_{12} \boldsymbol{\varepsilon}\right)}_{\mathrm{F}}+\mathbf{K}_{1} \mathbf{I}|\mathbf{s}|<0, \\
\mathbf{F}+\mathbf{K}_{1} \mathbf{I}|\mathbf{s}|<0 .
\end{gathered}
$$

From Eq. (20) it is clear that $\mathbf{K}_{1}$ must be negative, since $|\mathbf{s}|$ is always positive. After choosing $\mathbf{K}_{1}$, a negative value enough large to guarantee Eq. (20), Eq. (17) is satisfied and sliding mode occurs:

$$
\mathbf{s}=(\hat{\mathbf{i}}-\mathbf{i})=\dot{\mathbf{s}}=\mathbf{0} .
$$

After substituting Eq. (21) into the first raw of Eq. (16), it is now evident that:

$$
\mathbf{A}_{12} \varepsilon=-k_{1} \mathbf{I} \operatorname{sign}(\mathbf{s}) \text {. }
$$

We introduce a new switching function $\mathbf{z}=\left[z_{\alpha} z_{\beta}\right]$ as:

$$
\mathbf{z}=-k_{1} \mathbf{I} \operatorname{sign}(\mathbf{s}),
$$

which means that $z$ is a signal for compensating the back-EMF error $\varepsilon$, in Eq. (22).

By substituting the new switching signal Eq. (23) into the second raw of Eq. (16), the back-EMF error function in sliding mode condition is obtained (Chen et al., 2000; Freescale semiconductor, 2008):

$$
\dot{\varepsilon}=\left(\mathbf{A}_{22}-\mathbf{G} \mathbf{A}_{12}\right) \boldsymbol{\varepsilon} .
$$

The switching gain parameter design $k_{1}$ and the observer's pole assignment using $\mathbf{G}$ matrix can be found in Chen et al. (2000). The block diagram of the D-SMO is shown in Figure 4.

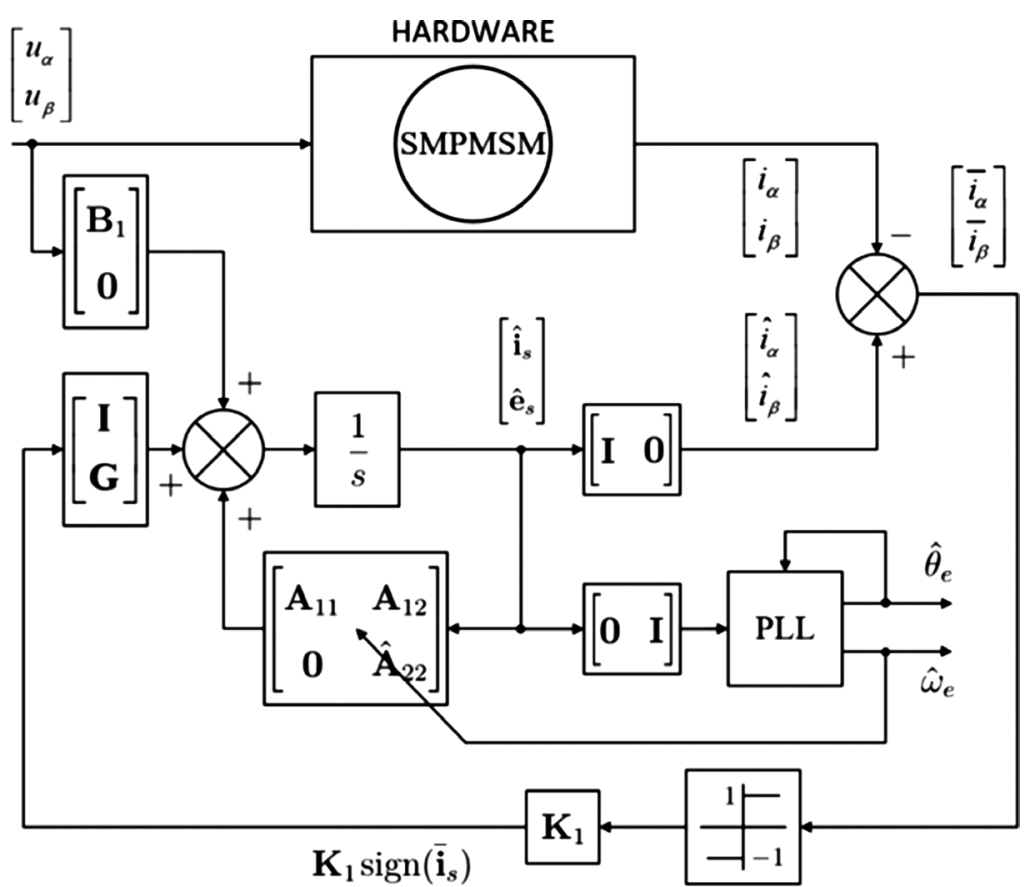

Fig. 4. The block diagram of direct SMO and PLL implementation. 


\section{Rotor Position Extraction from the Observed Back-EMF Components}

In many cases, the rotor position from the observed back-EMF components is obtained by using the arctangent function as follows:

$$
\hat{\theta}_{e}=\arctan \left(\frac{-\hat{e}_{\alpha}}{\hat{e}_{\beta}}\right) \text {. }
$$

It should be noted that the arctangent function is periodic between $[-\pi / 2, \pi / 2]$ and additional modification is needed before the observed electrical position $\hat{\theta}_{e}$ can be used in Park's transformations. Based on the equations in Section 2 for the back-EMF voltages, and according to vector diagrams of the back-EMF voltages along with the electrical position, it can be shown that for reversed rotation direction the observed position according to Eq. (25) needs to be shifted by a value of $\pi$. The estimated electrical angular speed can be calculated from the position using the following equation:

$$
\hat{\omega}_{e}=\frac{d \hat{\theta}_{e}}{d t} .
$$

Since arctangent function calculation Eq. (25) includes the division operation, the observation error of the rotor position may be amplified when the back-EMF values approach very low values. An advanced way to carry out the rotor position extraction is the use of the PLL circuit, as shown in Figure 5.

Its design can be found from examples in Wang et al. (2013). Parameters of the PI regulator in the PLL, $k_{p}$ and $k_{i}$ can be obtained from the following equations:

$$
\begin{aligned}
& k_{p}=2 \rho, \\
& k_{i}=\rho^{2}, \\
& \rho=\sqrt{\frac{a}{\Delta \theta_{e_{-} M A X}}} .
\end{aligned}
$$

The parameter $\Delta \theta_{e} \quad$ MAX is the user-defined expected maximum value of the observed position error.

The parameter $a$ can be calculated according to the following equation:

$$
a=\frac{p T_{d}}{J}
$$

where $p$ is the number of motor pole pairs, $T_{d}$ is the disturbance torque and $J$ is the moment of inertia. We calculated the value of the parameter $a$ from the nominal values of the motor. It can be seen in Eq. (28) that the value of

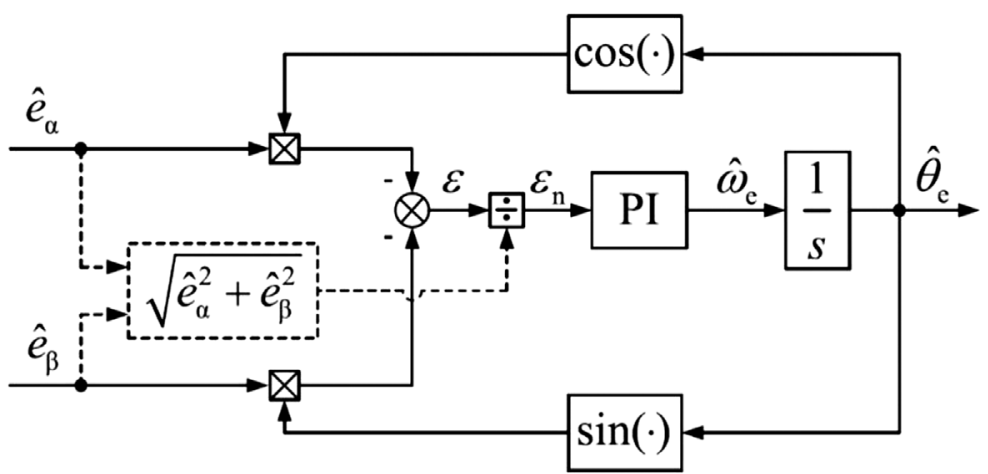

Fig. 5. Phase-locked loop based electrical position extraction (Wang et al., 2020). 
the parameter $a$ varies with the change of $T_{d}$ and $\mathrm{J}$. It should be noted that this variation may affect the overall performance of the PLL.

Since a low-pass filter (LPF) was used to obtain the observed back-EMF for the indirect observer (see Eq. (5)), a phase delay is introduced into the observed position. The phase delay in the observed position varies with current and motor voltage frequency, and it is highly desirable to compensate it. The two commonly used approaches for compensation are:

1. The filter cut-off frequency $\omega_{c}$ is always equal to the stator current frequency, which is related to electrical revolutions per second. A phase delay is fixed at $\pi / 2$ since the cut-off frequency varies with the rotor speed. The phase delay is then simply added to the observed rotor position and can be used in Park transformations (Zambada, 2010); or,

2. A fixed value of the cut-off frequency $\omega_{c}$ is used, and the phase delay is compensated according to the following equation (Haicai and Guangjun, 2016):

$$
\hat{\theta}_{e_{-} c o m p}=\hat{\theta}_{e}+\Delta \hat{\theta}_{e}=\hat{\theta}_{e}+\arctan \left(\frac{\hat{\omega}_{e}}{\omega_{c}}\right) \text {. }
$$

However, in the case of the direct SMO, the LPF is not used and the observed position calculated according to Eq. (25) or obtained by the PLL can be directly used without any phase delay compensation.

\section{Simulation Results}

The proposed indirect and direct SMOs were simulated using MATLAB/Simulink. The parameters of the machine and the parameters of both SMOs can be found in Table 1. The simulation results were obtained by using Universal Bridge with ideal switches and Permanent Magnet Synchronous Machine blocks from Simulink Simscape. Carrierbased sinusoidal PWM with a switching frequency of $20 \mathrm{kHz}$ was applied for simulation.

Different tuning parameters of the observers can be found in Table 1. The meaning of tuning parameters is different for both observers as the principles of their design are not the same. Consequently, in order to ensure a fair comparison between observers, their tuneable parameters were tuned with priority to the position error, which is the lowest steady-state error obtained between measured and estimated position.

For achieving a meaningful comparison results, the following conditions are set, which are the same for both observers. Observers have been implemented with standard field-oriented control. PI current controllers were designed for bandwidth $4 \mathrm{kHz}$ and PI speed controller was designed for bandwidth $40 \mathrm{~Hz}$. The sampling time of the current loop was $50 \mu$ s and sampling of the SMO observers was set to $10 \mathrm{~ms}$ to reduce the chattering effects. Otherwise, a detailed analysis of chattering and its reduction would be needed. Further, for both observers, the

Table 1. Parameters of the SMPMSM and tuning parameters for the SMOs

\begin{tabular}{|c|c|c|c|c|c|}
\hline \multirow{2}{*}{$\begin{array}{l}\text { Motor type } \\
\text { DC link voltage }\end{array}$} & \multirow{2}{*}{$\begin{array}{l}\text { TGN3-0115-30-48/T1 } \\
U_{D C}=48 \mathrm{~V}\end{array}$} & \multicolumn{2}{|c|}{ I-SMO parameters } & \multicolumn{2}{|c|}{ D-SMO parameters } \\
\hline & & $k_{1}$ & $75 \mathrm{~A} \cdot \mathrm{S}^{-1}$ & $\mathrm{k}_{1}$ & $-500 \mathrm{~A} \cdot \mathrm{S}^{-1}$ \\
\hline Rated torque & $M_{N}=1.13 \mathrm{Nm}$ & $f_{\text {cut-off }}$ & $600 \mathrm{~Hz}$ & $g_{1}$ & $-1.3 A^{-1}$ \\
\hline Rated current & $I_{N}=12.9 \mathrm{~A}$ & $|n| \leq 500 \mathrm{rpm}$ & $l=-0.1$ & $g_{2}$ & $0 A^{-1}$ \\
\hline Torque constant & $k_{t}=0.101 \mathrm{Nm} / \mathrm{A}$ & $|n|>500 \mathrm{rpm}$ & $l=1$ & & \\
\hline EMF constant & $k_{e}=6.12 \mathrm{~V} / 1000 \mathrm{rpm}$ & \multicolumn{4}{|c|}{ Low-pass filter for estimated speed filtering } \\
\hline Number of pole pairs & $p=5$ & $f_{L P}$ & $2000 \mathrm{~Hz}$ & & \\
\hline Rated speed & $n_{n}=3000 \mathrm{rpm}$ & \multicolumn{4}{|c|}{ PLL parameters } \\
\hline Stator resistance & $R_{2 p h}=0.258 \Omega$ & $\rho$ & 500 & & \\
\hline Stator inductance & $L_{2 p h}=0.6 \mathrm{mH}$ & $\Delta \theta_{e_{-} M A X}$ & $0.1 \mathrm{rad}$ & & \\
\hline Moment of inertia & $J=0.0002 \mathrm{~kg} \cdot \mathrm{m}^{2}$ & $T_{d}$ & $1 \mathrm{Nm}$ & & \\
\hline
\end{tabular}


same PLL was used for the rotor position extraction and the same low-pass filters were used for the final filtering of the estimated speeds.

Since the back-EMF is zero at standstill and has small values at low-speeds, the motor start-up is provided with a sensor in the closed-loop. After a predefined speed threshold, the switchover from measured to estimated speed (and position) is activated. The speed threshold was chosen as $200 \mathrm{rpm}$. In a real-time implementation, the PMSM start-up is usually done by an open-loop V/f or I/f method. It is noteworthy to mention that open-loop start-up has been applied in this paper neither for the purpose of simulation nor for that of experimental verification.

Figures 6 and 7 elucidate the performance of the speed and position estimation of the I-SMO during the simulation. The drive was loaded by a step change of the load torque with $80 \%$ of nominal value in $t=0.11-0.13 \mathrm{~s}$.
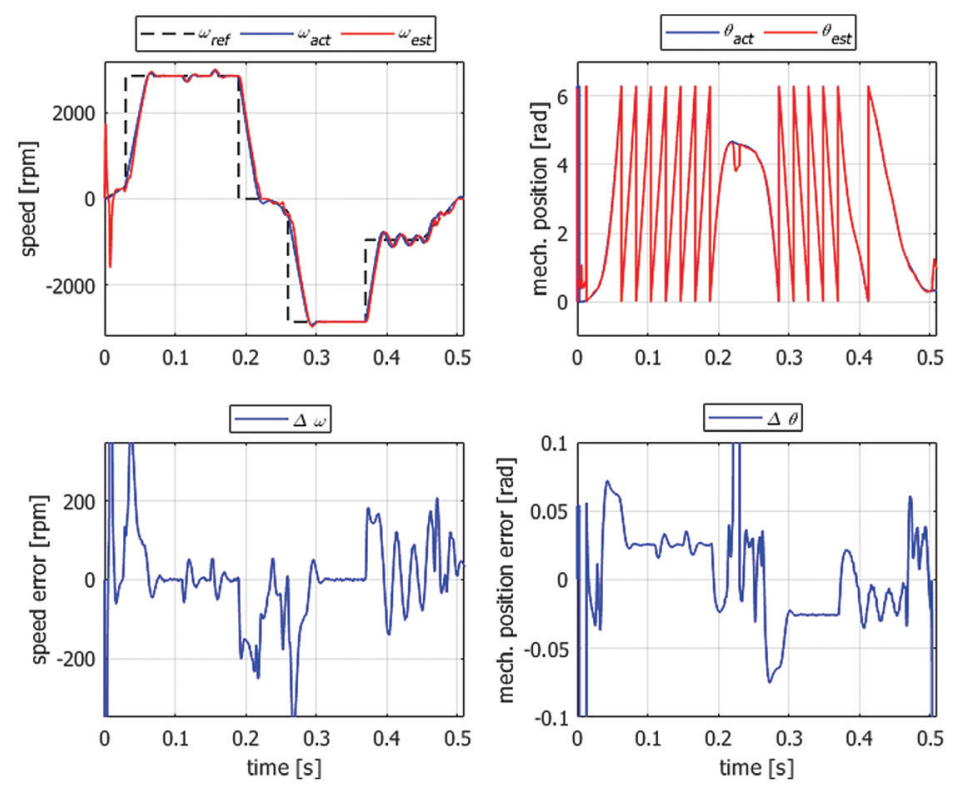

Fig. 6. I-SMO simulation: estimation of position and speed. The transition from sensored to sensorless operation switched for $n_{\text {act }}>200 \mathrm{rpm}$.
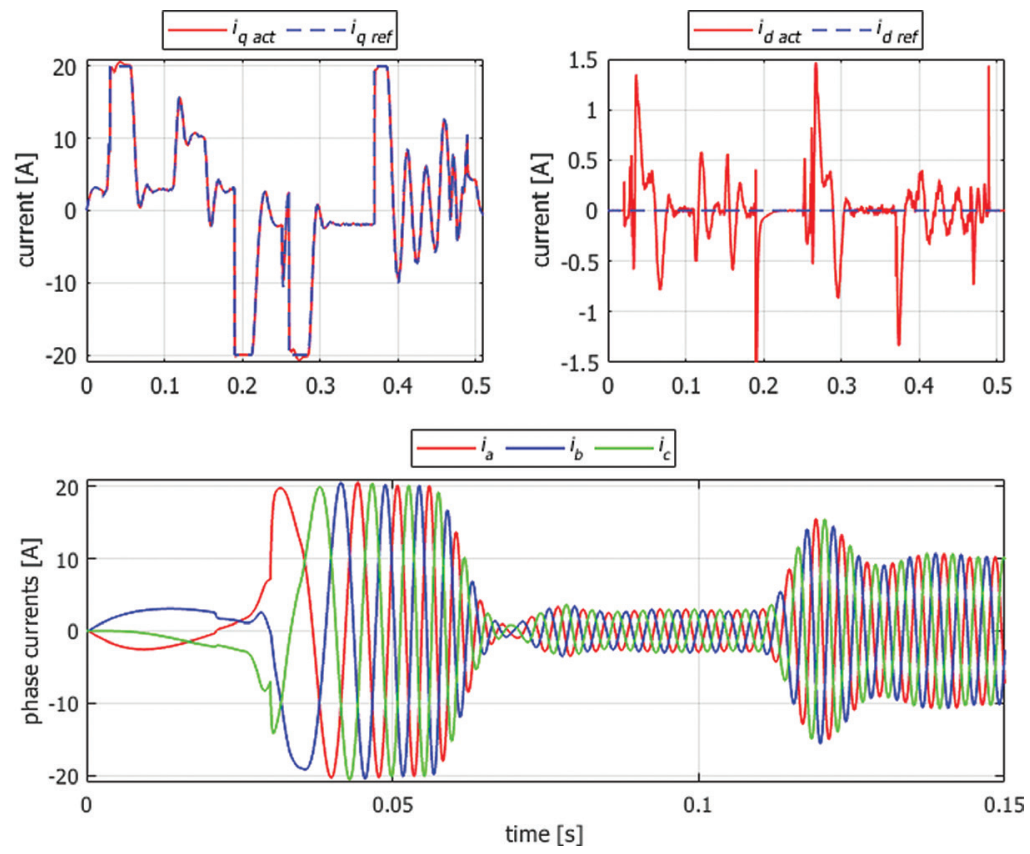

Fig. 7. I-SMO simulation: $d q$ and phase currents. The transition from sensored to sensorless operation switched for $n_{\text {act }}>200 \mathrm{rpm}$. 
The estimated speed generates high spikes during start-up and during low-speed operation, which is expected since the observed back-EMF components are small in this region. Spikes or incorrect values of observed rotor position can also appear in the low-speed region. The drive is operated in an open-loop (or with a sensor in our case) in this region; therefore neither the speed nor the position wrong estimates affect the performance of the drive.

It can be observed that speed estimation error is zero in steady-states and position estimation error in steadystate is around $0.025 \mathrm{rad}=1.43^{\circ}$. Position error estimation can be further reduced by an adjustment of the gain value $l$, but at a cost of higher speed oscillations.

Figure 7 shows decoupled control of $d$ and $q$ current components and smooth phase current response during sensorless control. The I-SMO gains were tuned experimentally because the result of the observer's stability analysis is only an interval of possible gain values in which the convergence of the observed to the real quantities is guaranteed.

Figures 8 and 9 elucidate the performance of the speed and position estimation of the D-SMO during the simulation. The drive was loaded by step change of the load torque with $80 \%$ of nominal value in $t=0.11-0.13 \mathrm{~s}$. As in the case of the I-SMO, the estimated speed generates high spikes during the start-up and the low-speed operation and this is again expected for the above-mentioned reasons. Further, the drive will be operated using feedback from the sensor for low speed, and therefore the wrong estimated speed values are not used in motor control. The same applies to the spikes in the observed position in the low speed region. Figure 9 shows decoupled control of $d$ and $q$ current components and smooth phase current response during sensorless control.

The D-SMO gains were tuned experimentally, and the priority was to get the lowest possible position error, which would result in position estimation error in a steady-state of $0.01 \mathrm{rad}=0,58^{\circ}$. The dynamics of the observer mostly depends on the choice of the $g_{1}$ value. This value is a compromise choice between the observer's convergence rate and the minimum speed value for the switchover from open-loop (or feed-back from the sensor in our case) to estimated speed feedback. To provide a fair comparison, this value was chosen to obtain the same switchover value for the D-SMO as for the I-SMO which was chosen as $200 \mathrm{rpm}$.

In Eq. (28), the calculation of the PLL parameters encompasses disturbance torque and moment of inertia values. In the paper, the disturbance torque $T_{d}=1 \mathrm{Nm}$ is constant. The total moment of machine inertia is often
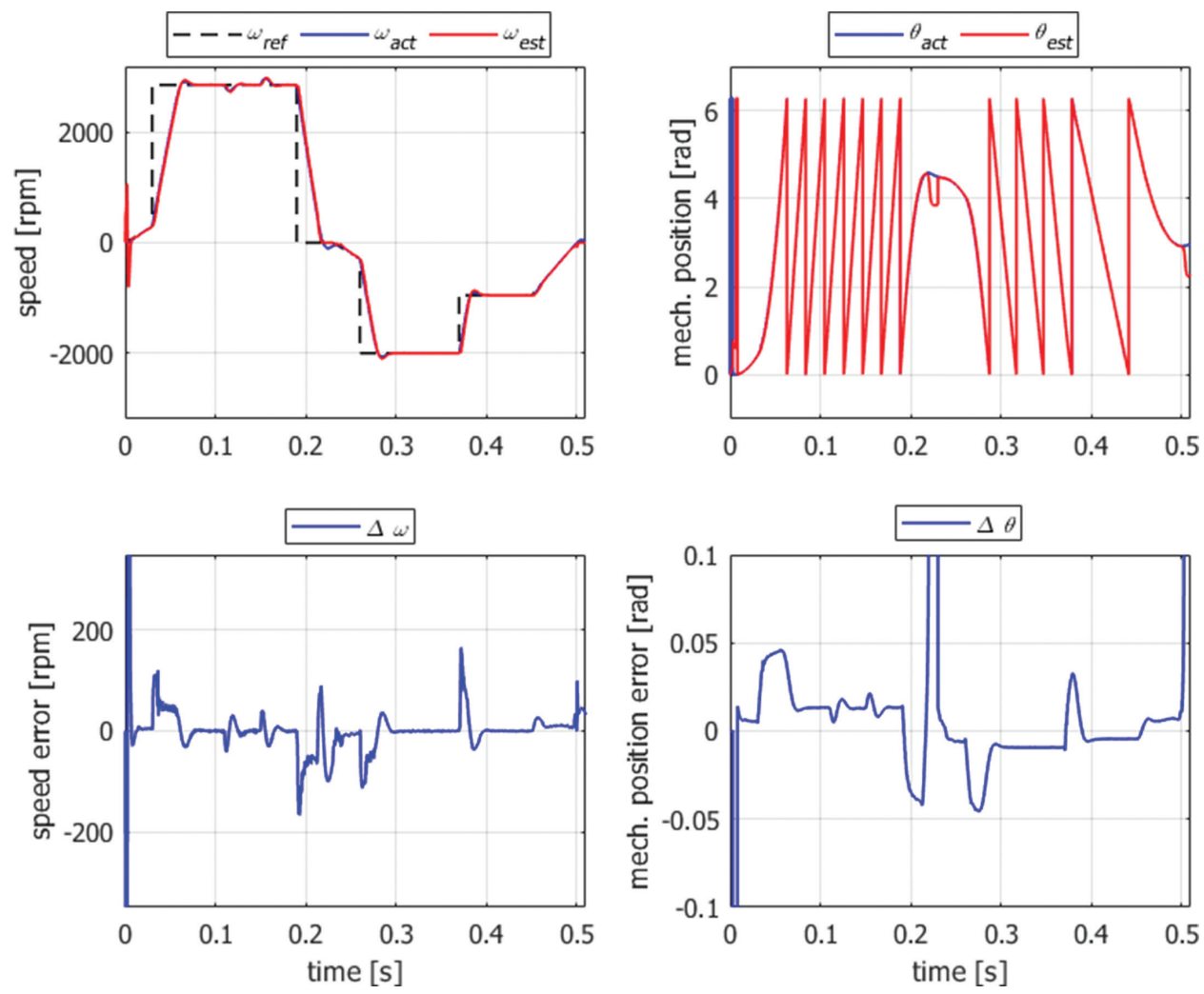

Fig. 8. D-SMO simulation: estimation of position and speed. The transition from sensored to sensorless operation switched in $n_{\text {act }}>200 \mathrm{rpm}$. 

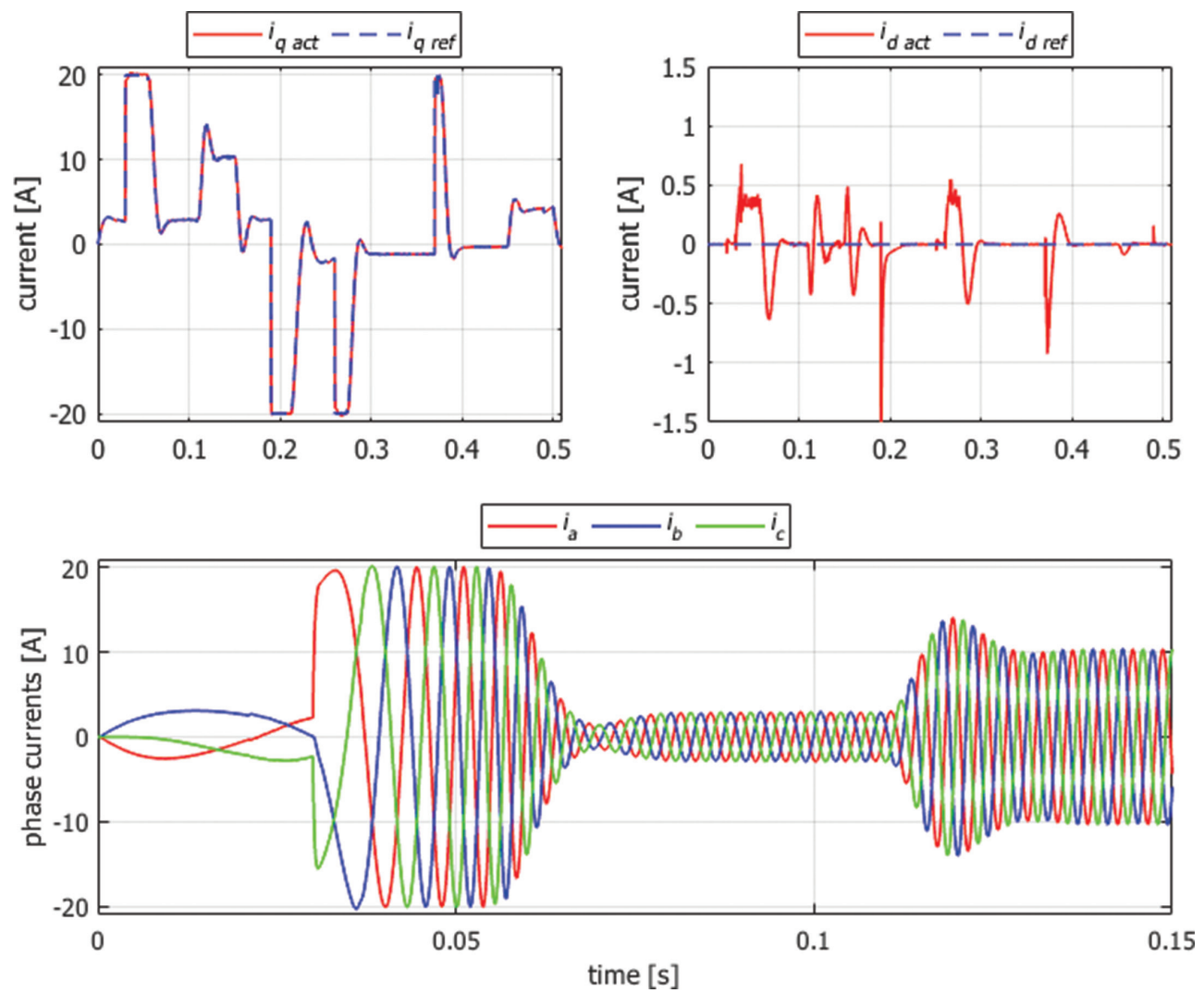

Fig. 9. D-SMO simulation: $d q$ and phase currents. The transition from sensored to sensorless operation switched for $n_{\text {act }}>200$ rpm.
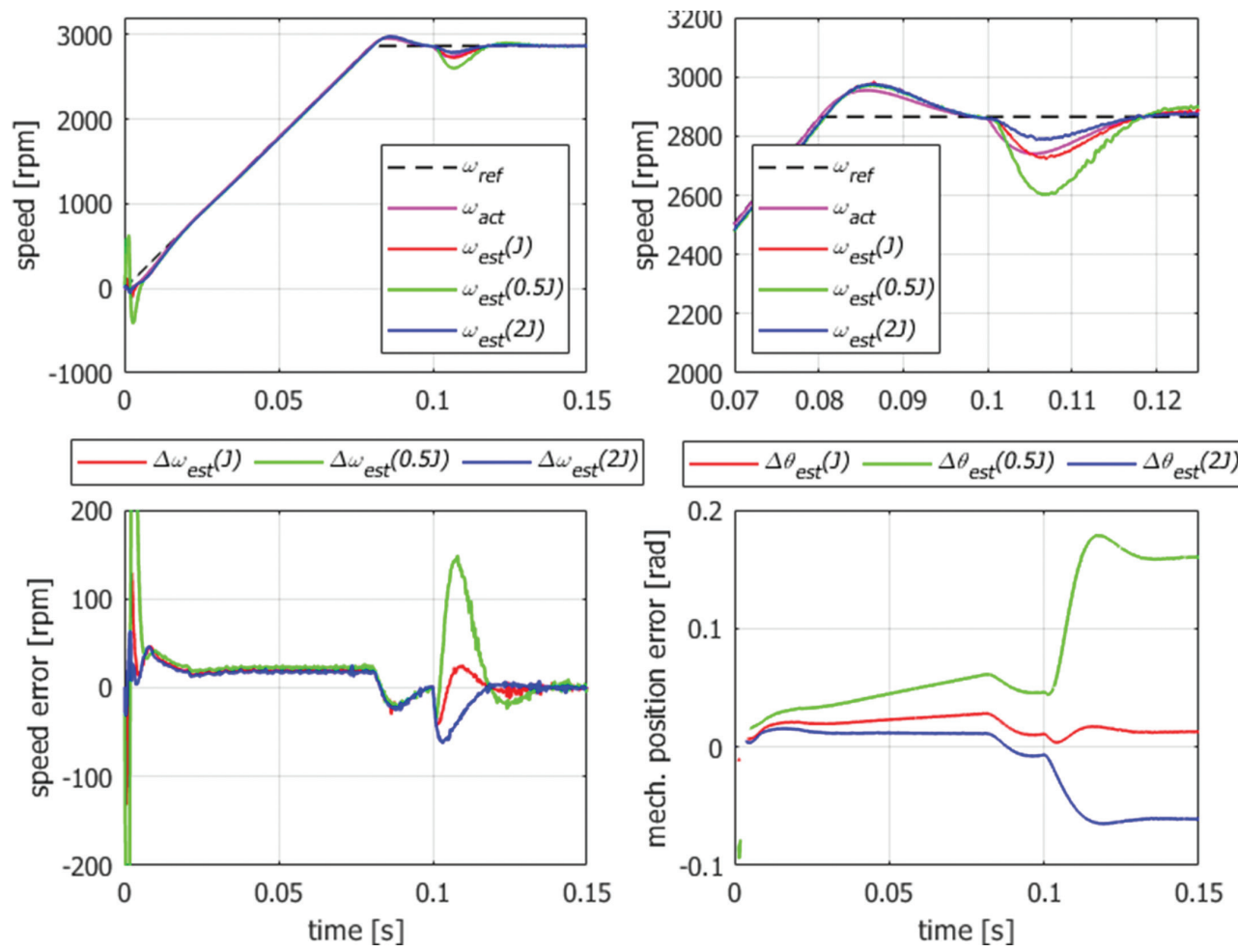

Fig. 10. Effects of the moment of inertia mismatches on the performance of the PLL. 
not known precisely and thus a mismatch between the real and calculated value can occur. Figure 10 shows the influence of this mismatch in position and speed estimation. The upper part of Figure 10 shows the time responses of the estimated speeds for inertia variation. The bottom right part of Figure 10 shows the influence of this variation on the position estimation error. The value of $J$ was changed in the mechanical equation of the machine whereas the value for the PLL (i.e. Eq. 28) remained unchanged. If the real inertia of the drive is two times higher (2J) or two times lower $(0.5 \mathrm{~J})$ against calculated inertia, then the speed and rotor position will be estimated with significant estimation error which occurs even in steady-state.

\section{Experimental Results}

The direct SMO has been chosen for experimental verification. The experimental setup consisted of an Opal RT 5600 hardware-in-the-loop simulator which is connected to a prototype of VSI, with integrated IRAM136-3023b VSI supplying SMPMSM with parameters given in Table 1. Incremental encoder with 1024 ppr was used for actual speed and position measurement. The sensorless control algorithm was developed with RT-LAB software and automated code generation was used to generate executive code for FPGA circuitry in OP 5600. Experimental results are shown in Figure 11.

Note that auxiliary algorithms are inevitable and have to be implemented for PMSM sensorless control, such as for initial position detection and open-loop start-up. In our case, a switchover from measured to estimated speed was used to simplify the observer implementation. Therefore, during low-speed operation ( $<200 \mathrm{rpm})$, no speed oscillations or repercussions are observed since the actual speed from the resolver is used as feedback. Figures 11(a) and 11(c) show overall speed performance and speed error, whereas Figures 11(b) and 11(d) show detailed speed and position error for speed transition from 50 to $100 \mathrm{rad} / \mathrm{s}$. The switchover between sensored and sensorless operation must be thoroughly provided in relation to the observer's tuneable parameters; otherwise, instabilities can occur. It can be observed in Figure 11 that the mean speed error is around $\pm 15 \mathrm{rpm}$ and the mean position error depends on the actual speed; further, for a given speed, which is around $8^{\circ}$; this is the same value usually found in the literature.

(a)

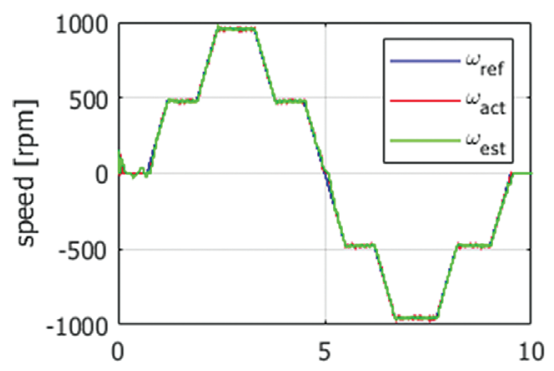

(c)

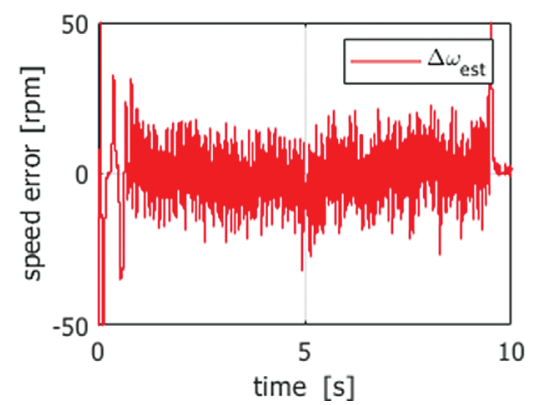

(b)

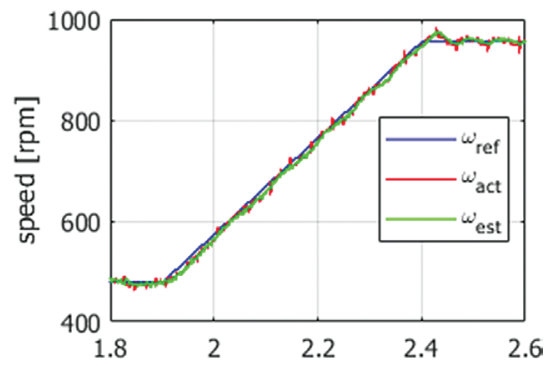

(d)

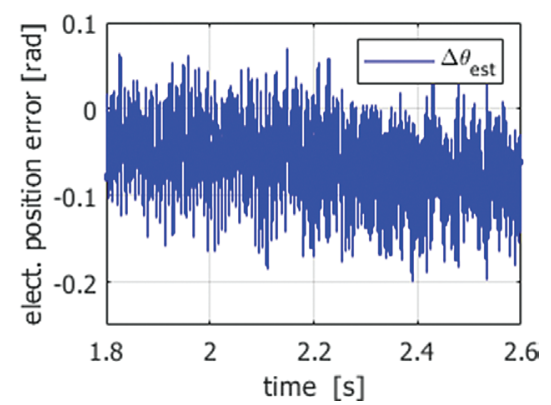

Fig. 11. D-SMO experimental verification. (a) ramp speed reference performance, (b) detail of the transition from 50 to 100 rad/s, (c) overall speed estimation error and (d) detailed position estimation error. 


\section{Conclusions}

Indirect and direct sliding mode observers for sensorless control of the SMPMSM machine were described and compared in this paper. The main objective of this work was to choose only one type that would be more suitable for experimental verification. The following conclusions are drawn from the comparison. The sampling time of both SMO algorithms must be chosen very carefully to avoid chattering effects, and it should be as low as possible considering DSP performance and computational resources. Simulation results show very similar performance for speed and position estimation for both observers and from this point of view, it does not matter which one should be chosen for experimental implementation. However, D-SMO has a complex mathematical description, but its tuning is more user friendly than for I-SMO. I-SMO has a simpler description and lower order, but its biggest disadvantage is that estimated back-EMF voltages must be filtered by the low-pass filter. This filtering causes considerable position delay that must be compensated afterwards. Further analysis is needed for parameter $l$ in speed adaptation of the I-SMO observer. Therefore, it is noteworthy to mention that more elaboration is needed for tuning and verification of the I-SMO.

\section{Acknowledgements}

This work was supported by the Scientific Grant Agency of the Ministry of Education of the Slovak Republic under the project VEGA 1/0493/19 and by the Slovak Research and Development Agency under Contract No. APVV-150750 .

\section{References}

Changsheng, L. and Elbuluk, M. (2002). A Robust Sliding Mode Observer for Permanent Magnet Synchronous Motor Drives. In: IEEE 2002 28th Annual Conference of the Industrial Electronics Society. IECON 02, Sevilla, 2002, vol. 2, pp. 10141019, doi: 10.1109/IECON.2002.1185411.

Chen, Z., Tomita M., Doki S. and Okuma, S. (2000). New Adaptive Sliding Observers for Position- and Velocity-Sensorless Controls of Brushless DC Motors. IEEE Transactions on Industrial Electronics, 47(3), pp. 582-591, doi: 10.1109/41.847899.

Chi, S. (2007). Position Sensorless Control of Permanent Magnet Synchronous Machines over Wide Speed Range. PhD. Dissertation, The Ohio State University.

Chi, S., Zhang Z. and Xu, L. (2009). Sliding-Mode Sensorless Control of Direct-Drive PM Synchronous Motors for Washing Machine Applications. IEEE Transactions on Industry Applications, 45(2), pp. 582-590, March-April 2009, doi: 10.1109/ TIA.2009.2013545.

Elbuluk, M. and Changsheng, L. (2003). Sliding Mode Observer for Wide-Speed Sensorless Control of PMSM Drives. In: 38th IAS Annual Meeting on Conference Record of the Industry Applications Conference, 2003., Salt Lake City, UT, USA, 2003, vol.1.1, pp. 480-485.
Freescale Semiconductor. (2008). Sensorless PMSM VectorControl with aSliding Mode ObserverforCompressors Using MC56F8013 [online]. Available at: https://www.nxp.com/docs/en/reference-manual/ DRM099.pdf [Accessed September 2008].

Haicai, L. and Guangjun, L. (2016). Rotor Position and Speed Estimation Method for Magnetically Suspended PMSM Based on Modified Sliding Mode Observer. In: 2016 International Conference on Mechatronics, Control and Automation Engineering, Bangkok, January 2016.

Horváth, K. and Kuslits, M. (2019). Speed Sensorless Field-Oriented Control of Induction Machines Using Unscented Kalman Filter. In: 2017 International Conference on Optimization of Electrical and Electronic Equipment (OPTIM) \& 2017 Intl Aegean Conference on Electrical Machines and Power Electronics (ACEMP), Brasov, 2017, pp. 523-528.

Kyslan, K., Šlapák, V., Petro, V., Marcinek, A. and Ďurovský, F. (2019). Speed Sensorless Control of PMSM with Unscented Kalman Filter and Initial Rotor Alignment. In: International Conference on Electrical Drives \& Power Electronics (EDPE), The High Tatras, Slovakia, 2019, pp. 373-378.

Tarczewski, T., Skiwski, M., Grzesiak, L. M. and Zieliński, M. (2018). PMSM Servo-Drive Fed by 
SiC MOSFETs Based VSI. Power Electronics and Drives, 3(38), pp. 35-45.

Texas Instruments. (2001). Digital Motor Control, Software library SPRU485A [online]. Available at: https://www.tij.co.jp/jp/lit/ug/spru485a/spru485 a.pdf?ts $=1601979782639 \&$ ref_url=https $\% 253$ A \%252F\%252Fwww.google.com\%252F [Accessed August 2020].

Utkin, V., Guldner, J. and Shi, J. (1999). Sliding Mode Control in Electromechanical Systems, 1st ed. Taylor \& Francis, London.

Wang, G., Li, Z., Zhang, G., Yu, Y. and Xu, D. (2013). Quadrature PLL-Based High-Order Sliding-Mode
Observer for IPMSM Sensorless Control with Online MTPA Control Strategy. IEEE Transactions on Energy Conversion, 28(1), pp. 214-224, doi: 10.1109/TEC.2012.2228484.

Wang, G., Zhang, G. and Xu, D. (2020). Position Sensorless Control Techniques for Permanent Magnet Synchronous Machine Drives. Springer: Singapore. ISBN 978-981-15-0049-7.

Zambada, J., Microchip Technology Inc. (2010). Sensorless Field Oriented Control of a PMSM. Microchip Application Note AN1078 [online]. Available at: http://ww1.microchip.com/downloads/ en/AppNotes/01078A.pdf [Accessed July 2020]. 